\title{
Improvising Design with a Haptic Instrument
}

\author{
Oliver S. Schneider* Karon E. MacLean ${ }^{\dagger}$ \\ Department of Computer Science \\ University of British Columbia, Vancouver, Canada
}

\begin{abstract}
As the need to deploy informative, expressive haptic phenomena in consumer devices gains momentum, the inadequacy of current design tools is becoming more critically obstructive. Current tools do not support collaboration or serendipitous exploration. Collaboration is critical, but direct means of sharing haptic sensations are limited, and the absence of unifying conceptual models for working with haptic sensations further restricts communication between designers and stakeholders. This is especially troublesome for pleasurable, affectively targeted interactions that rely on subjective user experience. In this paper, we introduce an alternative design approach inspired by musical instruments - a new tool for real-time, collaborative manipulation of haptic sensations; and describe a first example, mHIVE, a mobile Haptic Instrument for Vibrotactile Exploration. Our qualitative study shows that mHIVE supports exploration and communication but requires additional visualization and recording capabilities for tweaking designs, and expands previous work on haptic language.
\end{abstract}

Index Terms: H.5.2 [Information Interfaces and Presentation (e.g., HCI)]: User Interfaces-Haptic I/O

\section{INTRODUCTION}

Haptic feedback has hit the mainstream, present in smartphones, gaming and automobile design, but our knowledge of how to design haptic phenomena remains limited. There are still no agreedupon vocabularies or conceptual models for haptic phenomena $[15,21,23,26]$, in contrast to other modalities (e.g., using theory of minor chords to evoke a sad emotion in music). For subjective qualities, such as pleasant alerts or frightening game environments, prospects are even more limited. Design is still based on trial and error with programming languages, limiting exploration. The lack of established conceptual models or design frameworks further challenges communication between designers and stakeholders.

Using a music composition metaphor (as in [23]), we are writing music without ever playing a note. Instead, we compose a work in its entirety, then listen to the result before making changes. In contrast, musicians often use their instruments as a tool for serendipitous exploration when designing music and can draw upon musical theory. Furthermore, music is collaborative, with communication facilitated by a reference point of a sound. Touch, however, is a personal, local sense, making it difficult to discuss stimuli.

Facilitated exploration and collaboration should streamline the haptic design process and inform a guiding theory, analogous to those for musical composition. Designers will attain fluency with new devices and control parameters, while collaborative elements will get people designing in groups. A usable haptic language may emerge from their dialogue.

Our approach is to directly address these shortcomings with the development of a haptic instrument, inspired by musical instru-

*e-mail: oschneid@cs.ubc.ca

†e-mail: maclean@cs.ubc.ca

IEEE Haptics Symposium 2014

23-26 February, Houston, Tx, USA

978-1-4799-3131-6/14/\$31.00 @2014 IEEE

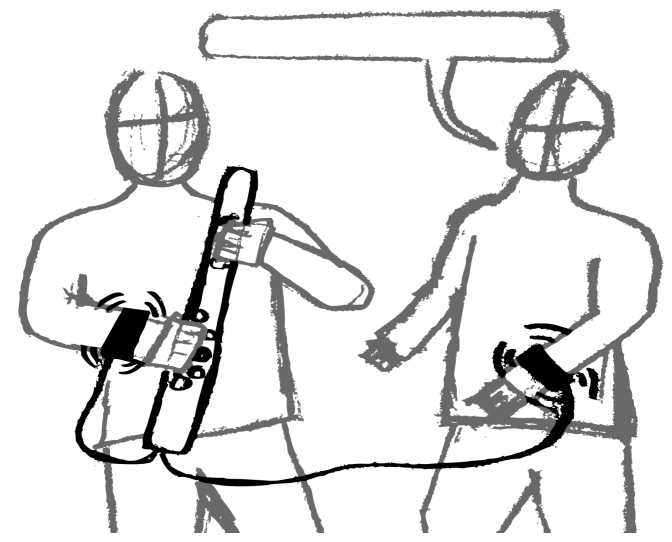

Figure 1: Concept sketch of a haptic instrument. Both users are experiencing the same sensation, controlled in real-time.

ments but producing (for example) vibrotactile sensations rather than sound (Figure 1). Haptic instruments have two main criteria: they provide real-time feedback to the user to facilitate improvisation and exploration, and produce haptic output to multiple users as a what-you-feel-is-what-I-feel (WYFIWIF) interface. This allows for a dialogue that includes a haptic modality: haptic instruments create a shared experience of touch, allowing for a common reference point. We developed a vibrotactile instance, mHIVE (mobile Haptic Instrument for Vibrotactile Exploration), as a platform to investigate this concept. Our main contributions are:

- A definition of the haptic instrument concept \& design space.

- A fully-working haptic instrument (mHIVE).

- The novel application of an established psychological methodology, phenomenology, to investigate mHIVE's interface and subjective tactile experiences.

- Preliminary results from a qualitative study that show mHIVE supports exploration and collaboration, and implications for the design of future haptic design tools.

In this paper, we first cover the related work of haptic design tools and haptic language, then define the haptic instrument, its requirements, features, and design space. We report the design of mHIVE, our methodology, and preliminary results, and conclude with future directions for haptic tool design and research into a haptic language.

\section{Related Work}

We cover previous work related to musical metaphors for haptic design, other tools for haptic design, and the language of haptics.

\subsection{Musical Metaphors in Haptic Design}

Musical analogies have frequently been used to inspire haptic design tools. The vibrotactile score, a graphical editing tool representing vibration patterns as musical notes, is an example of controlling vibrotactile (VT) sensations [22,23]. The vibrotactile score 


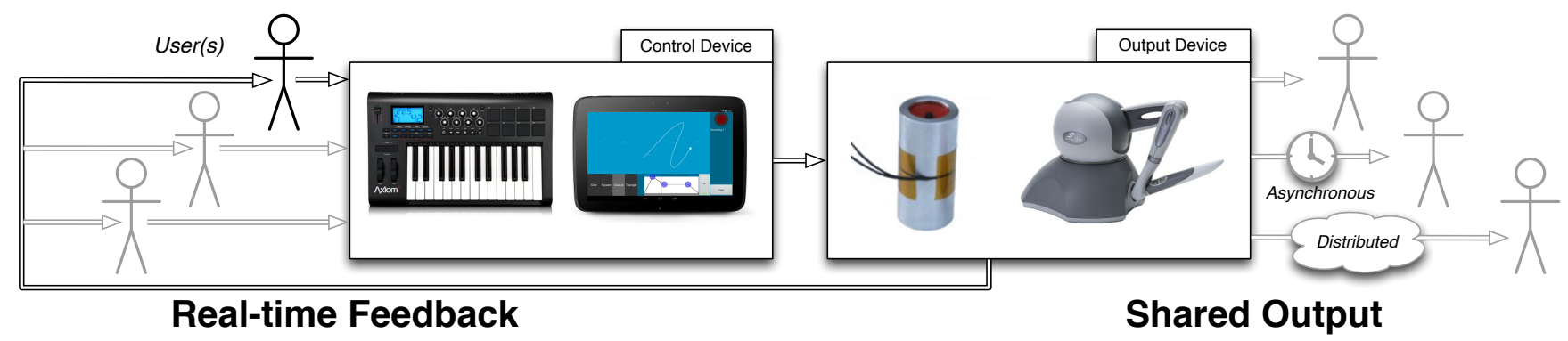

Figure 2: The haptic instrument concept. One or more people can control the instrument, and receive real-time feedback from the device. Any number of audience members can feel the output in real time as well. Control methods can vary, from traditional musical control devices (such as the M-Audio Axiom 25, used in preliminary prototypes) to touchscreen tablets (used in mHIVE). Output devices vary as well.

provides an abstraction beyond low-level parameters and can draw from a musician's familiarity with the notation, but we can take this idea further: when writing a song, a musician might improvise with a piano to try out ideas. We are inspired by the vibrotactile score and musical instruments, but define haptic instruments as a more general concept than literal musical instruments for touch.

Other musical metaphors include the use of rhythm, often represented by musical notes and rests $[6,7,9,32]$. Tactile analogues of crescendos and sforzandos have proven valuable to designing changes in amplitude [8]. Indeed, Brewster's original earcons and tactons were represented with musical notes $[4,5]$. The concept of a vibrotactile concert or performance was explored to identify relevant tactile analogues to musical pitch, rhythm, and timbre for artistic purposes [19]. As well, tactile dimensions have been used to describe or map to musical ideas [13]. Musical concepts have been widely used in the design of vibrotactile sensations, which we draw upon when designing mHIVE.

\subsection{Other Haptic Design Approaches}

Many tools have been developed to make it easier to work with the physical parameters of a haptic device. The Hapticon Editor is a graphical software tool that allows direct manipulation of the waveform for vibrations [15], and in another approach, piecing together of smaller iconic idioms [31]. This idea is best encapsulated by "haptic phonemes", the smallest unit of meaningful haptic sensations that can be combined [16]. A similar approach was used with TactiPEd, a graphical metaphor for control of wrist-based actuators, by controlling the low-level parameters of frequency, amplitude, and duration [28]. Haptic instrument parameters can be low- or high-level, but we use similar parameters with mHIVE.

Non-graphical approaches have also contributed to haptic design. Programming has benefitted from the use of toolkits such as HapticTouch, which uses higher-level descriptors ("Softness", "Breakiness") to control tangibles [21]. Though a promising direction, the vocabulary is not empirically grounded, and developers still have to deal with physical parameters. Hardware sketches and designing through making are also important approaches, since the immediate feedback of being able to feel haptics is crucial [24].

\subsection{Haptic Language}

Investigation into the language of tactile stimuli has a long history in psychological studies [27]. Many psychophysical studies have been conducted using factor analysis or similar approaches to determine the main tactile dimensions [27], but these have looked at materials rather than synthesized vibrotactile sensations, and have primarily been deductive (evaluating a pre-determined set of terms) rather than inductive (asking participants to describe sensations without prompting). Other work has shown little consensus on constant meanings for difference tactile dimensions, or whether a tac- tile language even exists [20]. There is a clear need to empirically investigate the subjective experience of touch-based interfaces, for which phenomenology is ideal $[11,25]$.

Our study is perhaps most closely related to Obrist, Seah, and Subramanian's work on the perception of ultrasound transducers [26]. Their study examined the language used to describe two different sensations, one oscillating at $16 \mathrm{~Hz}$ and the other at $250 \mathrm{~Hz}$. Though they also used phenomenology, our study differs in two important ways: we explore vibrotactile sensations rather than ultrasound, and give our participants a way of controlling the phenomenon directly, allowing for more coverage of the stimulus design parameters. A more deductive approach by Zheng and Morell also looked at how pressure and vibration actuators influenced affect, noting that affect influences attention, and documented qualitative descriptions of the sensations [35].

\section{Defining the Haptic InStrument}

We define a haptic instrument as a tool for general manipulation of one or more haptic (tactile, force-feedback, or both) devices that provides real-time feedback to anyone controlling the device, and can produce identical shared (WYFIWIF) output to all users to facilitate discussion and collaboration. Manipulation can include ideation, exploration, communication, recording, refinement, and articulation. Manipulation can be for utilitarian purposes (e.g., designing haptic notifications) or artistic expression (e.g., a haptic performance). Output devices can be purely output, or interactive. Furthermore, although haptic devices must be involved, multimodal experiences could easily be created by combining a haptic instrument with auditory or visual output. ${ }^{1}$

\subsection{Design Dimensions}

There are several main design dimensions that can be considered in a haptic instrument (outlined in Figure 2). A haptic instrument can occupy multiple positions on these dimensions.

Asychronous/synchronous. Though a haptic instrument must provide real-time feedback, its collaborative (shared-output) aspect could be either synchronous (by having multiple people experience the real-time output) or asynchronous (by allowing for recording and playback, important for design).

Collocated/distributed. A haptic instrument's output could be present only for users in the same room, or be broadcast over a network to people around the world. For example, multiple mobile devices could all display identical output in a distributed manner.

Private/shared control. A haptic instrument's control could be private (operated by a one person at a time) or shared (multiple users control the display). Shared control could be collocated or distributed (e.g., a web interface and shared object model).

${ }^{1}$ One could even imagine a multimodal instrument such as Asimov's Visi-Sonor [2] or its parody, Futurama's Holophonor [18]. 
Output mechanism. Each haptic instrument will control a haptic device, which has its own mechanism for providing a haptic sensation (e.g., vibrotactile sensations). Because haptic devices can be complex and combine multiple mechanisms, this is a large space in its own right. Characterizing the different display mechanisms is something that we must leave to future work. Suffice it to say, a haptic instrument will be different depending on its output device.

Number of haptic instruments or output devices. One consideration is whether a haptic instrument is intended to operate alone, or with other haptic/multimodal instruments. One can imagine haptic jam sessions for inspiration and ideation, or even form haptic bands for artistic expression. This is highly related to private/shared control - there is a fine line between several identical haptic instruments with private control, and a single haptic instrument with shared control and several output devices. Note that a haptic instrument may involve several devices to produce shared-output.

Control mechanism. Similarly, a haptic instrument could be controlled in a variety of ways. From musically-inspired MIDI controllers to smartphone applications, we envision a wide variety of control methods. Even a real-time programming environment might be appropriate for complex interactive sensations, so long as the control mechanism works with the output device's paradigm.

We expect that haptic instruments could provide both immediate and long-term value. We hope haptic instruments will improve the design process immediately, by supporting exploration and collaboration. Over time, their use could lead to a natural, emergent design language valuable in its own right. One can also imagine a general tool composed of several virtual haptic instruments, much like digital musical synthesizers.

\section{MHIVE}

We developed mHIVE to begin to explore how a haptic instrument should work and what it should do (Figure 3). mHIVE is collocated, synchronous, and mostly private in control; it accommodates shared display via dual Haptuators [34] and is operated with a single-touch tablet-based interface (Figure 4). We began with vibrotactile design because VT sensations are common, do not require interactive programming, are controlled through waveforms (analogous to music), and their low-level control parameters are well understood. A touchscreen allowed direct manual control.

mHIVE offers real-time control of frequency, amplitude, waveform, envelope, duration, and rhythm, identified as the most important parameters for vibrotactile sensations $[4,7,8,19,30]$.

The main view controls amplitude ( 0 to 1 ) on the vertical axis, and frequency $(0-180 \mathrm{~Hz}$, determined by piloting) horizontally. Amplitude and frequency were combined because we modeled them both as continuous controls: dynamics of continuous amplitude have been shown to be a salient design dimension $[8,19]$, and we did not want to choose discrete bins for frequency at this early stage. Further, single-handed control was essential - the other hand is required to feel the output. These axes were labeled to help users understand what they were and to give general sense of the values. A two-dimensional visual trace shows the previous two seconds of interaction history with the main view, intended to provide feedback and aid memory about drawings that were used.

VT duration and rhythm are directly mapped to screen-touch duration and rhythm. In analogy to musical timbre [4, 19], we provided four waveforms: sine, square, rising sawtooth and triangle. Sine and square are distinguishable [19], but we added sawtooth and triangle waveforms to expand the palette.

The attack-decay-sustain-release (ADSR) envelope controls amplitude automatically as duration of the note continues, as a 0-to1 multiplier of the amplitude displayed on the main amplitudefrequency input. Attack determines the amount of time (in milliseconds) to ramp the amplitude from 0 (none) to 1 (full). Decay determines the amount of time (in milliseconds) to ramp the amplitude

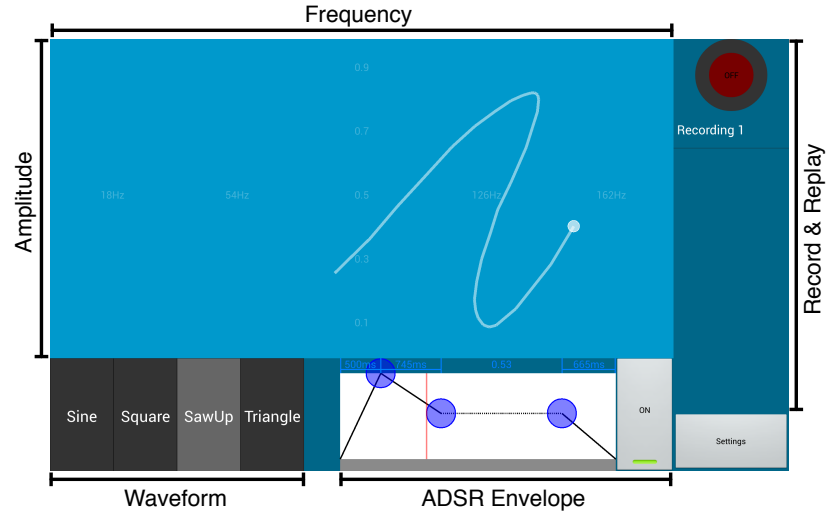

Figure 3: mHIVE interface. Primary interaction is through the amplitude-frequency view, where visual feedback is provided through a circle (current finger position) and a trail (interaction history).

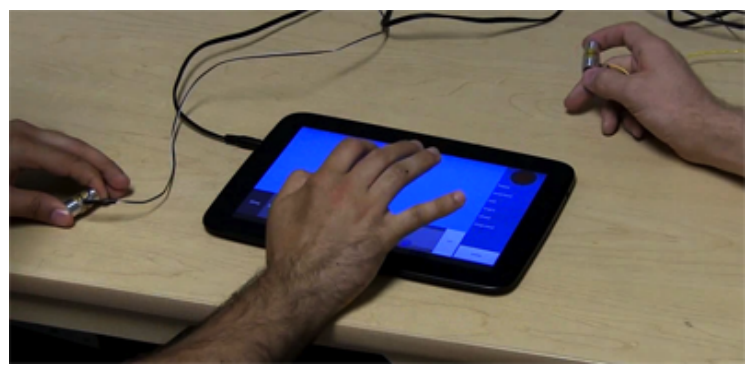

Figure 4: Study setup. Both the participant (left) and the interviewer (right) feel the same sensation as the participant controls mHIVE.

from 1 (full) to the sustain level. Sustain determines the amplitude level (from 0 to 1 ) held as long as the user keeps a finger on the display, playing a haptic note. Release determines the amount of time (in milliseconds) to ramp the amplitude from the sustain level to 0 (none). This envelope is a common feature of synthesized or digital musical instruments, and was noted as particularly useful in the Cutaneous Grooves project [19].

During piloting, we noticed that the ADSR concept was difficult to explain. We thus developed a novel interactive visualization, where the user could change the envelope parameters by dragging circles around. A red line operates as a cursor or playhead, showing the current progress through the envelope, looping around the dotted line when the sustain level is held.

Recording functionality was added to support more advanced rhythms and repetitions, and to allow users to save their sensations for later comparison. The record feature captures changes in frequency, amplitude, waveform, ADSR, and replayed recordings, allowing for compound haptic icons to be created. During playback, all changes are represented in the interface as if the user had manipulated them in real-time. At this time mHIVE only produces a single output sensation (with a single waveform, ADSR setting, frequency, and amplitude). Multitouch, layering, and sequencing (automatically playing multiple notes with a single touch) are not supported, as the semantics were too complex for a first design.

mHIVE is implemented in Java using the Android SDK [1], and the FMOD sound synthesis library [17] to produce sounds, sent to two or more Haptuators through an audio jack. We deployed mHIVE on an Android Nexus 10 tablet running Android 4.2.1. 


\section{Preliminary Study Methodology}

We conducted a preliminary qualitative study to investigate two questions. First, is mHIVE an effective tool for the expression, exploration, and communication of affective phenomena? Second, what language, mental models, and metaphors do people use to describe vibrotactile sensations, and how do they relate to mHIVE's low-level control parameters?

We collected and analyzed our data using the methodology of phenomenology, an established variant of qualitative inquiry used in psychology to investigate topics ranging from visual illusions to tactile experience [11,26,29]. Phenomenology explores subjective experience, appropriate for an investigation into the more intangible qualities of pleasantness and affect. At this point, the rich, inductive data of qualitative analysis is more valuable than a controlled experiment with statistical analysis.

In particular, we use the Stevick-Colaizzi-Keen method as described by Moustakas [25]. In-depth interviews are conducted with a small number of participants. The interviewer, Researcher 1 (R1), also documents his experience, as if he was interviewing himself. Then, R1 transcribes each interview, including his own. Transcripts are divided into non-overlapping, non-redundant statements about the phenomena known as Meaning Units (MUs). This considers every statement that the participants make, and does not discount any due to bias or selective searching. Then, MUs are clustered into emergent themes. We interpret our themes in the Discussion.

\subsection{Procedure}

Our 1-hour open-ended interviews used the following protocol:

1. Ask the participant for their background: occupation, experience with touchscreens, haptics, music, and video games.

2. Demonstrate mHIVE to the user, and invite them to explore while thinking aloud to describe the sensations they feel.

3. Probe the design space by asking participants to explore different control parameters, and to explore their metaphors (e.g., if the participant describes a sensation as "smooth", R1 would ask them to try to produce a "rough" sensation).

4. Ask the participants to produce sensations for the six basic cross-cultural emotions documented by Ekman [14], and rank how well they think their sensation represents the emotion on a 4-point semantic differential scale (Very Poorly, Somewhat Poorly, Somewhat Well, Well). This was done both as an elicitation device to gather a wider range of interactions with mHIVE, and to directly investigate a design task.

5. Set the Haptuators down, and ask the participants to describe their experience of working with mHIVE in as complete detail as possible to evaluate the device itself.

$\mathrm{R} 1$ conducted the interviews and analysis, which required specialized knowledge of mHIVE. Scores of inter-rater reliability common with other qualitative analyses (e.g., grounded theory [10]) are inappropriate and unavailable, as we did not conduct deductive, lowlevel coding. To improve reliability, R1's documented experience was analyzed first, and then consulted during analysis to remove bias (e.g., to not use terms only used by the experimenter).

\section{Results}

We sought participants with experience designing haptics as a proxy for expert designers for our initial study. Four participants were recruited through email lists and word-of-mouth (P1-4, three male), and were all in the age range of 26-35 with self-reported occupations including graduate students or post-docs in information visualization, HCI, and human-robot interaction). All had experience working with haptic technology, and (because of this requirement) all knew the main researcher in a professional capacity, although only P2 had seen earlier prototypes of the haptic instrument. The small sample size, typical for phenomenological studies [11], was appropriate for the rich data we wanted. Data collection ended when we achieved saturation of new results, and had a clear direction for our next iteration.

Here we report the three major themes that emerged during analysis: mHIVE's success as a haptic instrument, mHIVE's limitations that reveal more detail about the haptic design process, and the use of language in the study.

\section{1 mHIVE Succeeds as a Haptic Instrument}

Our results suggest that mHIVE can be effective for exploration of a design space, and communication in the haptic domain. Overall, mHIVE was well received, seen as a novel and promising tool. "I definitely liked it" (P1), "I think there should be more devices like this for designing haptic icons" (P2).

Serendipitous exploration. Participants reported that mHIVE was best served to explore the design space, generate a number of ideas, and try things out. Serendipitous discoveries and exclamations of surprise were common. Participants were able to "accidentally stumble upon something" (P2) as they explored the device. "I felt I could get a large variety", "I could easily play around with the high-level to find out what was neat" (P3).

Communication. mHIVE established an additional modality for dialogue. The dual outputs created a shared context, demonstrated by deictic phrases: the additional context of the vibrotactile sensation was required to make sense of the statement. The use of "that" and "there", reminiscent of the classic "Put That There" multimodal interaction demo [3] indicate a shared reference point was established from the haptic instrument. "So there'd be like, (creates a sensation on the device), which is pretty mellow" (P3).

In particular, P4 successfully communicated the sensation of sleepiness to the R1, by asking whether R1 could guess the sensation. "Can you guess it?" (P4) "Sleepy?" (R1) "Yeah. Pretty good" (P4). The dialogue worked as a two way channel, as R1 was able to phrase questions using the device. "It was different" (P2) "How was it different?" (R1) "You delayed the first part, it felt new" (P2).

Certain sensations, like a feeling of randomness, could only be felt when another person controlled mHIVE. "When someone else does it, I feel better, it's like, you cannot tickle yourself” (P2).

\subsection{Tweaking through Visualization and Modification}

During analysis, some key directions for future design emerged around visualization and control capabilities.

Inability to tweak. Though mHIVE supported exploration and collaboration, we found it was inadequate as a standalone design tool. Few created sensations were considered to be final. Many descriptions were hedged and in the design task, few sensations captured the emotional content well. "I dunno, maybe that's afraid?" (P1), "Still felt that you can make them better" (P2), "To me that's more fuming (laughing) than it is angry" (P3). On some occasions, participants were certain about their descriptions. "Sad, definitely down on the amplitude with sad... oh that's totally sad. Yeah.” (P1). This was uncommon, and usually tied to discovering an ideal sensation during the design task.

More visualization and recording. Part of mHIVE's inability to support tweaking was due to cognitive limitations for both memory and attention. Participants found it difficult to remember what they had tried before, and to pay attention to the output while simultaneously controlling it. "There's a lot of variables which, when I'm trying to compare between two configurations... it was hard sometimes to remember what I had tried" (P3), "I definitely liked being 
able to feel a stimulus without having to implement it, you know, it allows me to focus more on what it feels like" (P1).

Participants suggested that although visualization and recording features helped somewhat to overcome these limitations, more was needed. All requested greater emphasis on recording through repetition or looping, both to aid memory and allow for focus on the sensation independent of device control.

Allowing persistent, modifiable sensations and alternative visualizations could also help participants overcome these limitations. "The recording records what I do, but it'd be nice to have it repeat stuff" (P3), "It might conceivably be nice to be able to, you know, draw a curve, draw a pattern, draw like you would in paint, and then be able to manipulate it, replay it, move the points, see what happens" (P1).

\subsection{A Difficult Language}

Our study was too small to analyze language patterns in detail, but exposes emerging trends.

Pleasantness, ADSR, and frequency. Participants often started with a statement of like or dislike rather than a description. Pleasant sensations often involved the ramp-in and ramp-out ("echo" or "ringing") of the ADSR envelope, or lower-frequency sensations. Longer, higher frequency without ramp-in and ramp-out were less pleasant. "I don't know how else to describe it, I kinda like it" (P1), "Yeah, this [ADSR] seems natural, somehow", "It feels unnatural to kill the echo right away" (P2), "I like this [low-frequency] sensation cuz to me it feels a lot like purring” (P3).

Waveform. Participants all noticed differences between waveforms, but were often challenged in expressing them (P4 used the musical term "timbre"). Square waves in particular were distinct, with a greater range and stronger affinity to mechanical sensations. "It's interesting, they feel more different than I thought they would" (P1), "If you want to make something feel like a motorcycle, you would definitely need square wave" (P2).

Aural/haptic metaphors drawn from previous experience. For the most part, participants used concrete examples and direct analogies to describe sensations, often drawn from their previous experiences. One stand-out strategy employed by all participants was onomatopoeias: "beeooo" (P1\&4), "vroom” (P1), "bsheеeоoo", "boom”, "nеeеaa", "mmmMMMmmm" (P2), "pa pa pa pa”, "tum tum tum tum", "tumba tumba tumba tumba" (P3); "upward arpeggio, like, (singing with hand gestures) na na na naaa" (P4). Other sound-based metaphors were very common, including hum, buzz, whistle, rumble (P1); bell (P1, P2); squeaky, creak (P2); or thumpy (P3). Still other descriptors were directly haptic in nature: rough, flat (P1); sharp, round, ticklish (P2); sharp, smooth, cat pawing (P3); impatient foot tapping (P4).

\section{Discussion}

Here we interpret these themes to draw implications for haptic design tools, and compare to research on the language of haptics. We then reflect upon our methodology and limitations.

\subsection{Design Tools}

mHIVE was able to achieve the two main goals of a haptic instrument, facilitating both exploration and collaboration. Participants were clearly able to explore the different low-level parameters, and encountered serendipitous or unexpected sensations through improvisation. mHIVE created a shared experience that facilitated communication between R1 and the participants. We can thus conclude that haptic instruments are a promising new tool in a haptic designer's arsenal, with a first, successful implementation in mHIVE.

However, the second theme shows that serendipity and communication are only part of the equation. mHIVE does not serve as a general editor of haptic sensations. In particular, participants found their attention split when controlling the device and feeling the sensation; perhaps the real-time control should allow for a rapid, but not instantaneous, switch in focus between control and perception. More generally, participants were unable to tweak sensations because there was insufficient support for comparing ideas or evolving an existing idea.

In hindsight, this general difficulty is understandable given the broader context of the musical instrument analogy we used for inspiration. Musical instruments are not used to write songs on their own, but combined with notation or recording media. A similar combination of a haptic instrument and recording might be described more succinctly as a haptic sketchpad. Sketching is critical in design because it allows for the evolution of an idea through multiple sketches, as well as criticisms, comparisons, and modifications [12]. Emphasizing a history feature that supports multiple versions of sketches, the user could develop an idea as if with a multiple pages in a sketchbook. Haptic sketching in hardware has already been shown to be effective [24]. As well, a visual metaphor resonates with the desire for more effective visualization.

Ultimately, haptic instruments may be most useful as one element in a suite, or component of a more general tool. A haptic instrument could complement a graphical editing tool that does support tweaking, such as the vibrotactile score $[22,23]$ or the hapticon editor [15]. As part of a more comprehensive tool, mHIVE could be improved to reduce cognitive barriers to memory and attention. Alternatively, we could add functionality to mHIVE to support looping, visualization, and direct manipulation of the sensations within the tool. We will explore these options as we iterate on mHIVE's design in future work.

\subsection{Language}

Our preliminary results for language are compatible with the literature, supporting previous work. Participants' readiness to say whether a sensation was pleasant or not supports the view that touch is affective in nature, and that knowing what one likes or doesn't like is a primary function of touch [20]. ADSR pleasantness and high-frequency unpleasantness are both consistent with the literature: Zheng and Morell note that ramped signals influenced affect more positively than step signals, and 3 s high-frequency sensations were annoying or agitating [35]. The heavy use of onomatopoeias is reminiscent of Watanabe et al.'s work with static materials [33]. However, in our study, onomatopoeias were often used to express dynamic sensations (beeeoo being a gradual decrease in amplitude and frequency), which might be a useful direction for future work.

\subsection{Methodology and Limitations}

Although phenomenology is uncommon in the haptics community (excluding [26]), we found it to be an effective way to empirically examine the subjective experience of using mHIVE. Because the community is still developing processes and tasks for haptic design, qualitative studies seem to be an especially appropriate way to tackle these problems. Once we have further defined haptic design, we can then move to more task-based, experimental methods.

Our study was a first round of feedback to inform our next iteration, and has limitations. First, our participant pool is (intentionally) small, and participants were all collected through our professional network, as people with haptic design experience are rare. As we continue to tackle the problem of haptic design, we hope to seek out a larger and more diverse pool of participants, and explore more realistic design tasks.

\section{CONCLUSION}

In this paper, we have introduced the concept of the haptic instrument, a new tool for haptic designers that supports serendipitous exploration and collaboration. We described the implementation of mHIVE, a mobile Haptic Instrument for Vibrotactile Exploration, 
with design decisions drawn from the literature. Our findings suggest that haptic instruments are effective tools for improvised exploration and collaboration, but only support part of the design process. Additional tools or features are required to support tweaking. Finally, we reported the use of language when interacting with mHIVE, expanding upon several conclusions in the literature.

We believe this to be a step towards a greater goal, the establishment of haptic design as its own discipline, with processes, tools, and best practices. Future work will build on this base as we continue to examine the haptic design process. We will consider a haptic sketchpad concept as one way to overcome the cognitive barriers, and allow users to tweak their designs. We also hope to apply haptic instruments and other tools in more realistic design scenarios. By supporting designers at this critical point, we can continue to make haptics more valuable than ever.

\section{ACKNOWLEDGMENTS}

This work was supported by NSERC and approved by UBC's Behavioural Research Ethics Board (\#H13-01620). We are very grateful to our participants.

\section{RefERENCES}

[1] Android Open Source Project. Android Developers, 2012.

[2] Asimov, I. Foundation and Empire. Gnome Press, 1952.

[3] Bolt, R. A. Put-that-there: Voice and Gesture at the Graphics Interface. ACM SIGGRAPH Computer Graphics 14, 3 (July 1980), 262 270.

[4] Brewster, S., and Brown, L. M. Tactons: structured tactile messages for non-visual information display. In 5th Australasian User Interface Conference (AUIC '04) (Jan. 2004), 15-23.

[5] Brewster, S. A., Wright, P. C., and Edwards, A. D. N. An evaluation of earcons for use in auditory human-computer interfaces. In Proceedings of the SIGCHI conference on Human factors in computing systems (CHI '93), ACM Press (New York, USA, May 1993), 222227.

[6] Brown, L., Brewster, S., and Purchase, H. A First Investigation into the Effectiveness of Tactons. In First Joint Eurohaptics Conference and Symposium on Haptic Interfaces for Virtual Environment and Teleoperator Systems, IEEE (2005), 167-176.

[7] Brown, L. M., Brewster, S. A., and Purchase, H. C. Multidimensional tactons for non-visual information presentation in mobile devices. In Proceedings of the 8th conference on Human-computer interaction with mobile devices and services (MobileHCI '06), ACM Press (New York, USA, Sept. 2006), 231-238.

[8] Brown, L. M., Brewster, S. A., and Purchase, H. C. Tactile crescendos and sforzandos. In Extended abstracts on human factors in computing systems (CHI EA '06), ACM Press (New York, USA, Apr. 2006), 610615.

[9] Chan, A., MacLean, K., and McGrenere, J. Designing haptic icons to support collaborative turn-taking. International Journal of HumanComputer Studies 66, 6 (2008), 333-355.

[10] Corbin, J., and Strauss, A. Basics of Qualitative Research: Techniques and Procedures for Developing Grounded Theory, 3 ed. Sage Publications, Inc., 2008.

[11] Creswell, J. W. Qualitative inquiry and research design: choosing among five approaches, 3rd ed. Sage Publications Inc, 2013.

[12] Cross, N. Design Thinking: Understanding How Designers Think and Work. Berg Publishers, Oxford, UK, 2011.

[13] Eitan, Z., and Rothschild, I. How music touches: Musical parameters and listeners' audio-tactile metaphorical mappings. Psychology of Music 39, 4 (Nov. 2010), 449-467.

[14] Ekman, P., Davidson, I. R., Ellsworth, P., and Friesen, W. V. Are There Basic Emotions? Psychological Review 99, 3 (1992), 550-553.

[15] Enriquez, M., and MacLean, K. The hapticon editor: a tool in support of haptic communication research. In 11th Symposium on Haptic Interfaces for Virtual Environment and Teleoperator Systems, 2003 (HAPTICS '03), IEEE Comput. Soc (2003), 356-362.

[16] Enriquez, M., MacLean, K., and Chita, C. Haptic phonemes: basic building blocks of haptic communication. In Proceedings of the 8th international conference on Multimodal interfaces (ICMI '06), ACM Press (New York, USA, Nov. 2006), 302.

[17] FMOD. FMOD Ex API, 2013.

[18] Futurama. Parasites Lost, 2001.

[19] Gunther, E., Davenport, G., and O'Modhrain, S. Cutaneous grooves: composing for the sense of touch. In Proceedings of the 2002 Conference on New Instruments for Musical Expression (NIME '02) (May 2002), 1-6.

[20] Jansson-Boyd, C. V. Touch matters: exploring the relationship between consumption and tactile interaction. Social Semiotics 21, 4 (Sept. 2011), 531-546.

[21] Ledo, D., Nacenta, M. A., Marquardt, N., Boring, S., and Greenberg, S. The HapticTouch toolkit. In Proceedings of the Sixth International Conference on Tangible, Embedded and Embodied Interaction (TEI '12), ACM Press (New York, USA, Feb. 2012), 115-122.

[22] Lee, J., and Choi, S. Evaluation of vibrotactile pattern design using vibrotactile score. In IEEE Haptics Symposium (HAPTICS '12), IEEE (Mar. 2012), 231-238.

[23] Lee, J., Ryu, J., and Choi, S. Vibrotactile score: A score metaphor for designing vibrotactile patterns. In Third Joint EuroHaptics conference and Symposium on Haptic Interfaces for Virtual Environment and Teleoperator Systems (World Haptics '09), IEEE (Mar. 2009), 302 307.

[24] Moussette, C., and Banks, R. Designing through making. In Proceedings of the fifth international conference on Tangible, embedded, and embodied interaction (TEI '11), ACM Press (New York, USA, Jan. 2011), 279-282.

[25] Moustakas, C. Phenomenological Research Methods. SAGE Publications, Inc., 1994.

[26] Obrist, M., Seah, S. A., and Subramanian, S. Talking about tactile experiences. In Proceedings of the SIGCHI Conference on Human Factors in Computing Systems (CHI '13), ACM Press (New York, USA, 2013), 1659-1668.

[27] Okamoto, S., Nagano, H., and Yamada, Y. Psychophysical Dimensions of Tactile Perception of Textures. IEEE Transactions on Haptics 6, 1 (Jan. 2013), 81-93.

[28] Paneels, S. A., Anastassova, M., and Brunet, L. TactiPEd: Easy Prototyping of Tactile Patterns. INTERACT 20138118 (2013), 228-245.

[29] Richer, P. A phenomenological analysis of the perception of geometric illusions. Journal of Phenomenological Psychology 8, 2 (1978), 123135.

[30] Rovan, J., and Hayward, R. Typology of Tactile Sounds and their Synthesis in Gesture-Driven Computer Music Performance. Trends in Gestural Control of Music (2000), 297-320.

[31] Swindells, C., Maksakov, E., MacLean, K., and Chung, V. The Role of Prototyping Tools for Haptic Behavior Design. In 14th Symposium on Haptic Interfaces for Virtual Environment and Teleoperator Systems (HAPTICS '06), IEEE (2006), 161-168.

[32] Ternes, D., and MacLean, K. E. Designing Large Sets of Haptic Icons with Rhythm. Lecture Notes in Computer Science 5024 (2008), 199208.

[33] Watanabe, J., Hayakawa, T., Matsui, S., Kano, A., Shimizu, Y., and Sakamoto, M. Visualization of Tactile Material Relationships Using Sound Symbolic Words. In Haptics: Perception, Devices, Mobility, and Communication, P. Isokoski and J. Springare, Eds., vol. 7283 of Lecture Notes in Computer Science. Springer, Berlin, Heidelberg, 2012, 175-180.

[34] Yao, H.-Y., and Hayward, V. Design and analysis of a recoil-type vibrotactile transducer. The Journal of the Acoustical Society of America 128, 2 (Aug. 2010), 619-627.

[35] Zheng, Y., and Morrell, J. B. Haptic actuator design parameters that influence affect and attention. In 2012 IEEE Haptics Symposium (HAPTICS), IEEE (Mar. 2012), 463-470. 